\title{
The role of ketogenic diets in the therapeutic management of adult and paediatric gliomas: a systematic review
}

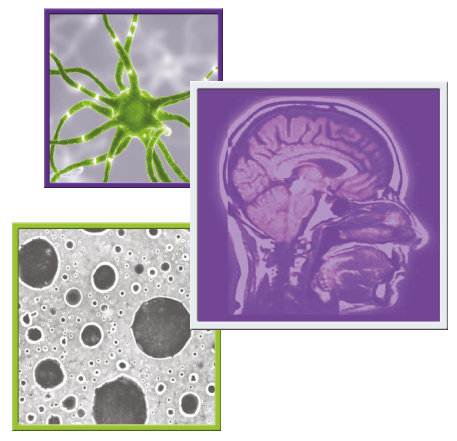

\author{
Kirsty J Martin-McGill*.1, Nisaharan Srikandarajah ${ }^{1}$, Anthony G Marson ${ }^{1}$, Catrin Tudur \\ Smith $^{2}$ \& Michael D Jenkinson ${ }^{1}$ \\ ${ }^{1}$ Institute of Translational Medicine, University of Liverpool \& The Walton Center NHS Foundation Trust, Lower Lane, Liverpool L9 \\ 7LJ, UK \\ ${ }^{2}$ Department of Biostatistics, University of Liverpool, Brownlow Hill, Liverpool L69 3BX, UK \\ *Author for correspondence: kirsty.martin@liverpool.ac.uk
}

\section{Summary points}

- This article systematically reviews the evidence for the effectiveness and acceptability of different ketogenic diets (KD) in the therapeutic management of patients with gliomas.

- Six studies have been published since 1995, conducted in the USA and Europe $(n=39)$.

- All studies are case series evidence, the lowest position in the evidence hierarchy. However, at present this is the only evidence available to inform decisions regarding the implementation of KDs for gliomas.

- Minimal adverse events were reported, suggesting KDs to be safe in this population.

- The evidence for effectiveness and acceptability of various KDs is insufficient to suggest they have a therapeutic effect in the management of gliomas; therefore further high-quality research is needed.

- Key areas for future research include:

- A pragmatic feasibility study to inform future RCT design.

- High quality randomized control trials to determine if KDs are effective in the management of glioma.

- A health economic assessment to establish efficiency, clinical effectiveness and value of the intervention.

Aim: Weperformed a systematic review of the evidence for effectiveness and acceptability of different ketogenic diets (KDs) in the therapeutic management of gliomas. Methods: The search strategy included searches of seven electronic databases. Data extraction and quality assessment were undertaken independently by two authors. Results: No randomized clinical trials were identified. Six studies $(n=39)$ met the eligibility criteria for this review - all were case series or reports and therefore at high risk of bias. All studies reported overall or progression-free survival; however the effectiveness of KD interventions could not be established. Dietary acceptability was not reported. Conclusion: The effectiveness and acceptability of KDs in the management of gliomas is unknown and high quality randomized controlled trials are needed.

First draft submitted: 22 January 2018; Accepted for publication: 31 January 2018; Published online: 16 April 2018

Keywords: glioblastoma • glioma • ketogenic diet • systematic review

\section{Description of the condition}

Primary brain tumors affect 7.14 per 100,000 of the worldwide population each year [1], with gliomas being the commonest form of malignant brain tumor, affecting three to five people per 100,000 each year [2]. Despite current treatment options including surgical resection, radiotherapy and chemotherapy, these tumors remain incurable and the prognosis is poor.

\section{Description of the intervention}

Warburg first recognized that tumor cells rely on glucose for energy in 1926 [3]. Over the decades the Warburg theory has been developed further, leading to the hypothesis that switching gliomas' energy source from glucose to ketones may result in cancer cell death [4]. One mechanism for achieving this is by targeting tumor energy 
Table 1. A PICO table illustrating the systematic review question and criterion.

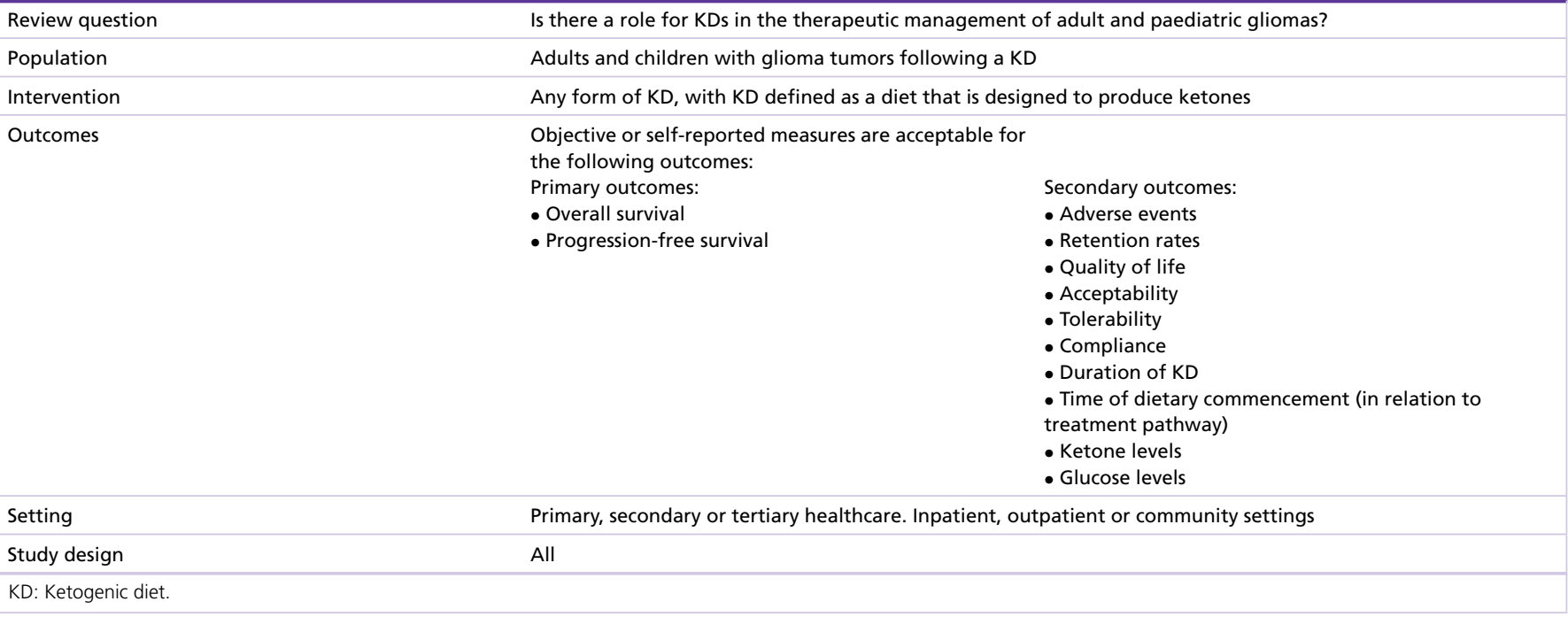

metabolism with the ketogenic diet (KD); a high fat, low carbohydrate diet, which results in the production of ketones as a primary energy source, while minimizing glycolysis through glucose restriction [5].

$\mathrm{KDs}$ are perceived as unpalatable and their assessment in patients with poor survival outcomes has therefore been limited. However, following the success of the classic 4:1 KD ( $4 \mathrm{~g}$ fat for $1 \mathrm{~g}$ of carbohydrate and protein combined, $\sim 90 \%$ total energy from fat) in pediatric epilepsy, dietary variants have been developed that are designed to be more palatable and easier to implement, with fewer side effects [6].

Why this review is important?

A systematic review and meta-analysis of the antitumor effects of $\mathrm{KD}$ in mice demonstrated a prolonged survival for the $\mathrm{KD}$ groups compared with standard diet (mean survival time ratio $=0.85[95 \%$ highest density interval $=$ $0.73,0.97]$; hazard ratio $=0.55$ [95\% highest posterior density interval $=0.26,0.87]$ ) [7]. To our knowledge, no such review exists for human studies and a review of the best current evidence is required to inform decisions about service provision and the design of future clinical trials.

Research question \& aim

Aim: to review the evidence for effectiveness and acceptability of different KDs in the therapeutic management of patients with gliomas.

\section{Methods}

The protocol for this systematic review was registered with PROSPERO (identification number: CRD42017056752).

The population, intervention, comparison and outcomes (PICO) table below illustrates the review question and inclusion criterion (population, intervention, outcomes, setting and study design). As all study designs were considered, a comparator arm was not essential (see Table 1).

No restriction was placed on year of study or publication status. The search was limited to English language publications.

\section{Search strategy}

A four-part search strategy was implemented to identify suitable studies.

\section{Electronic searches}

The following electronic databases were searched.

- EMBASE

- PubMed 
- Cochrane Library

- CINAHL Plus

- MEDLINE

- SCOPUS

- Web of Science

The primary search was undertaken on 25 January 2017, with updates identified until 18 August 2017 (see the Supplementary Appendix for an example search strategy).

\section{Hand searches}

References of the included studies were hand searched to identify other possible studies.

\section{Study registries}

The following study registries were searched:

- ClinicalTrials.gov

- The World Health Organisation International Clinical Trials Registry Platform

- UK Clinical Trials Gateway

- International Standard Randomized Controlled Trial Number Register (ISRCTN)

- National Institute of Health Clinical Trials Registry

- National Research Register Projects Database Achieve

- PROSPERO

The search was undertaken on 21 March 2017, with updates identified until 18th August 2017.

\section{Other resources}

Conference abstracts and posters were included in the search to identify recent studies undertaken that may not be published or are ongoing.

\section{Screening of included studies}

Duplicate references were removed from the search results. Prepiloted inclusion criteria were applied to titles and abstracts identified in the search results. Full text was obtained for studies identified for potential inclusion and inclusion criteria were reapplied. Following this, an expert in the field (AC Scheck) was also contacted to identify any studies that were shortly due for completion or publication.

\section{Reporting results of searches}

The Preferred Reporting Items for Systematic Reviews and Meta Analyses (PRIMSA) flow diagram was adopted to document the number of references located from databases and other sources, the number of duplicates removed, records screened, records excluded, full text articles screened, full text articles excluded with reasons and the number of studies included in the final review [8].

\section{Data extraction \& quality assessment}

Two authors (KJ Martin-McGill and N Srikandarajah) independently extracted data using a prepiloted data extraction form. Any discrepancies were discussed between the two authors in the first instance. A third author (MD Jenkinson) was available for consultation if disagreements would have occurred.

Although all study types were permitted for inclusion in the review, only case studies and case series were identified. Therefore the Institute of Health Economics (Canada) Case Series Quality Appraisal Checklist was selected as the appropriate quality assessment tool [9]. Again, the tool was applied by KJ Martin-McGill and N Srikandarajah. 


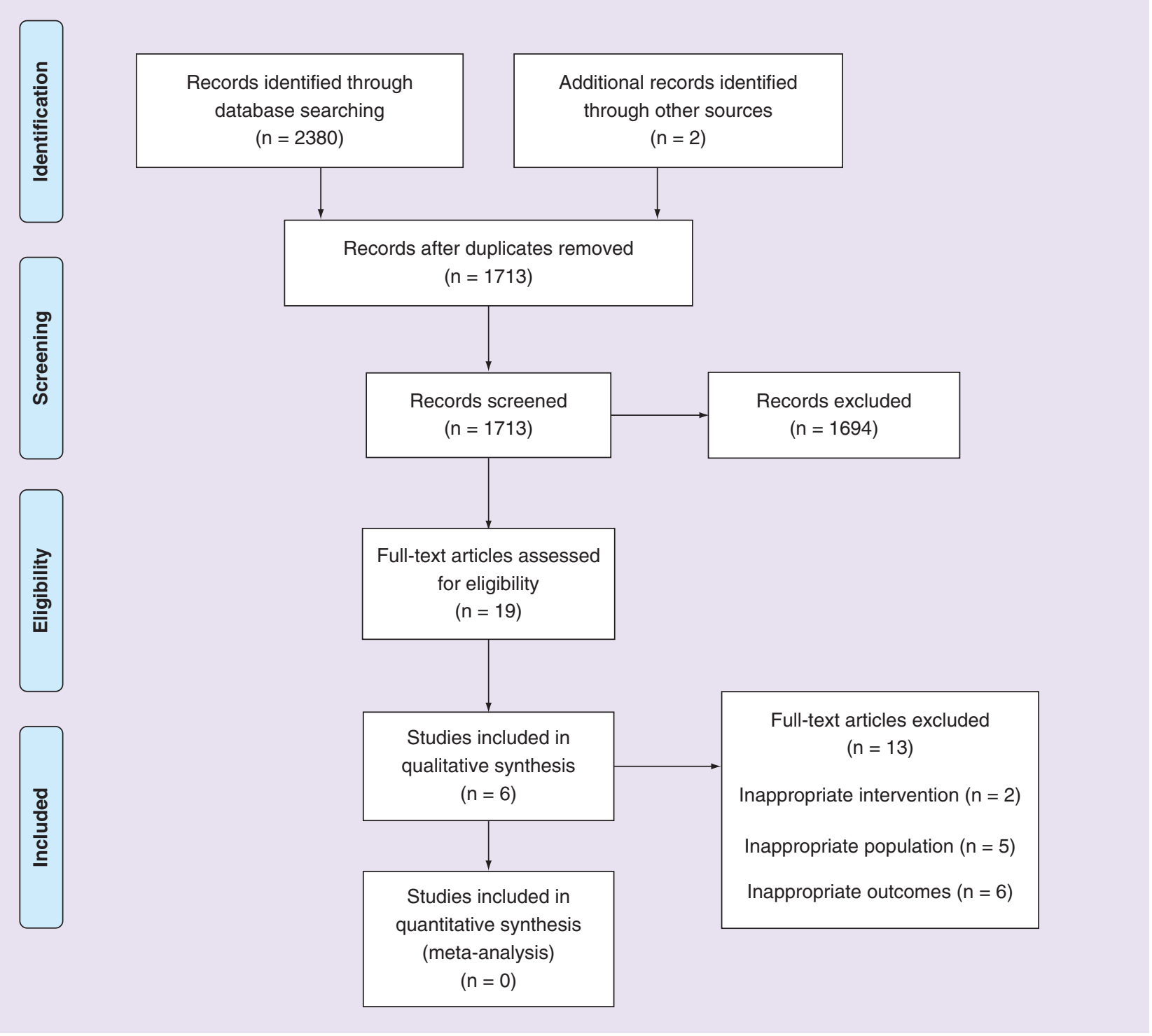

Figure 1. PRISMA flow diagram.

Adapted with permission from [8] Moher et al. (2009).

\section{Results \\ Description of studies \\ Results of the search}

The electronic search identified 2380 records, and another two were identified by searching the references of included studies. After removing duplicates, 1713 records remained. Following the screening of titles and abstracts, 19 remained eligible for inclusion. These studies underwent a full text review, following which a further 13 studies were excluded due to inappropriate interventions [10,11], inappropriate populations [7,12-15] and inappropriate outcome measures [16-20]. Therefore six studies met the eligibility criteria for this review (Figure 1) [21-26]. No further studies were identified from the expert in the field.

\section{Included studies}

Six studies have been published since 1995, conducted in the USA and Europe. Population sample size varied between one and 20 participants, with a variety of KD interventions. Table 2 provides a summary of the study characteristics. 


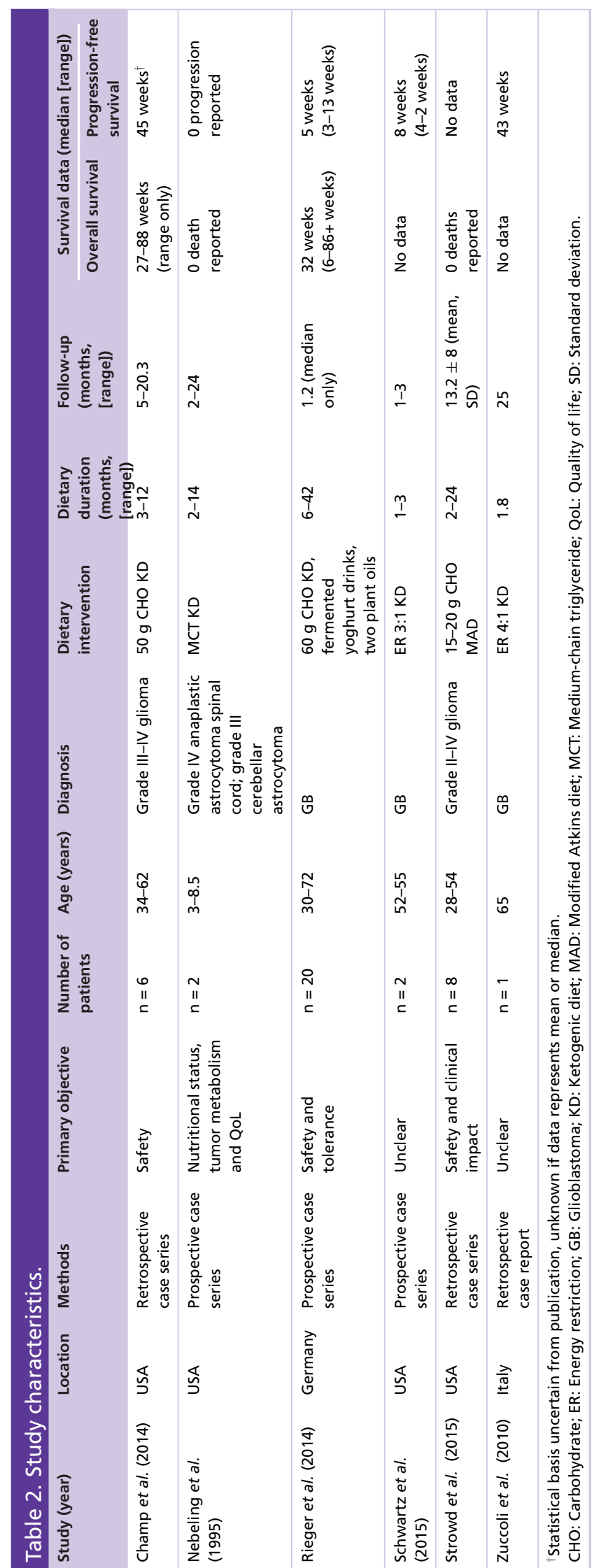


Risk of bias in included studies

All six studies were at high risk of bias and a summary of the quality assessment can be found in Table 3 .

\section{Effects of interventions \\ Critical outcomes \\ Overall survival}

Four studies reported overall survival (OS; $\mathrm{n}=36$ ). Follow-up ranged from 6 to 91 weeks. Two studies comprising ten patients reported no deaths [23,26]. Two studies comprising of 22 patients reported survival ranging from 6 to $88+$ weeks [21,22]. Survival can be related to diagnosis and dietary intervention in Table 2.

\section{Progression-free survival}

Five studies reported progression-free survival (PFS; $\mathrm{n}=30$ ). Time to progression ranged from 3 to 45 weeks in four studies [21-22,24-25]. One study comprising of two patients reported no progression following 62 weeks on diet, at the time of writing $(n=1)$; however, PFS for the second patient was not reported [26]. PFS can be related to diagnosis and dietary intervention in Table 2.

\section{Important outcomes}

Adverse events

All studies reported adverse events $(n=39)$. The most frequently reported adverse effects related to KD interventions were weight loss [21-25], ranging from $-2.2 \%$ body weight [22] to $-13 \%$ body weight [25] and increased cholesterol [24,26]. Other adverse effects reported in low numbers were deep vein thrombosis [21], grade III leukopenia [22], lymphopenia [25], hyperuricemia [25], hypoproteinemia [26].

\section{Dietary retention rates}

The retention rate could be determined for three studies $(n=24)$, all undertaken prospectively using a defined protocol, which ranged from 50 to $100 \%$ [22,24,26]. Retention was determined at 8 weeks $(n=2)$ [26], 12 weeks $(n=2)$ [24] or at the point of tumor progression $(n=20)$ [22] (median PFS 5 weeks, range 3-13 weeks). Reasons for withdrawal from diet included tumor progression $(n=1)[24]$ and negative impact on quality of life $(n=3)$; however no validated tool was documented [22].

\section{Quality of life}

No studies reported quality of life using appropriate objective or subjective measures.

Acceptability

No studies reported dietary acceptability using appropriate objective or subjective measures.

\section{Tolerability}

Two studies reported dietary tolerability $(\mathrm{n}=18)$. Grade I constipation was reported at dietary initiation $(\mathrm{n}=2)$, grade I fatigue $(\mathrm{n}=4)$ during radiotherapy and grade II fatigue $(\mathrm{n}=1)$ during $30 \%$ energy restricted $30-50 \mathrm{~g}$ carbohydrate $\mathrm{KD}$ [21]. Gastrointestinal assessment reported diarrhoea at a mean intensity of $<1$ (weak), constipation at a mean intensity of $<1$ (weak), hunger of mean intensity of $>1$ but $<2$ (weak to moderate) and demand for glucose mean intensity of $>1$ but $<2$ (weak to moderate), using a nonvalidated questionnaire $(n=12)$ [22].

\section{Compliance}

Three studies reported dietary compliance $(n=24)$. Maintenance of ketosis was used as a surrogate for compliance in two studies [24,26], while patient self-reporting demonstrated compliance for 6.8 days per week $(n=20)$ [22].

Ketone levels

Five studies reported ketosis $(n=24)$. Three studies reported serum ketosis $(n=6)$, with levels between levels of $0.3 \mathrm{mmol} / \mathrm{l}$ (indicates reported units have been converted to $\mathrm{mmol} / \mathrm{l}$ from $\mathrm{mg} / \mathrm{dl}$ for comparison) to $7 \mathrm{mmol} / \mathrm{l}$ $(\mathrm{n}=4)$ [21,24] and maintenance of serum ketosis was reported by one study $(\mathrm{n}=2)$ [26]. Urinary ketosis was reported by two studies $(\mathrm{n}=14)$. One study reported urinary ketones between 1.5 and $2.5 \mathrm{mmol} / \mathrm{l}$ during the first 3 weeks of $\operatorname{diet}(\mathrm{n}=1)$ [25]. In the other study, urinary ketosis achieved at least once in $92 \%$ participants $(n=12 / 13)$ and when assessing all urinary measurements from 12 participants, ketonuria was present in $73 \%$ of cases [22]. Methodology and frequency of testing was not consistent between studies. 


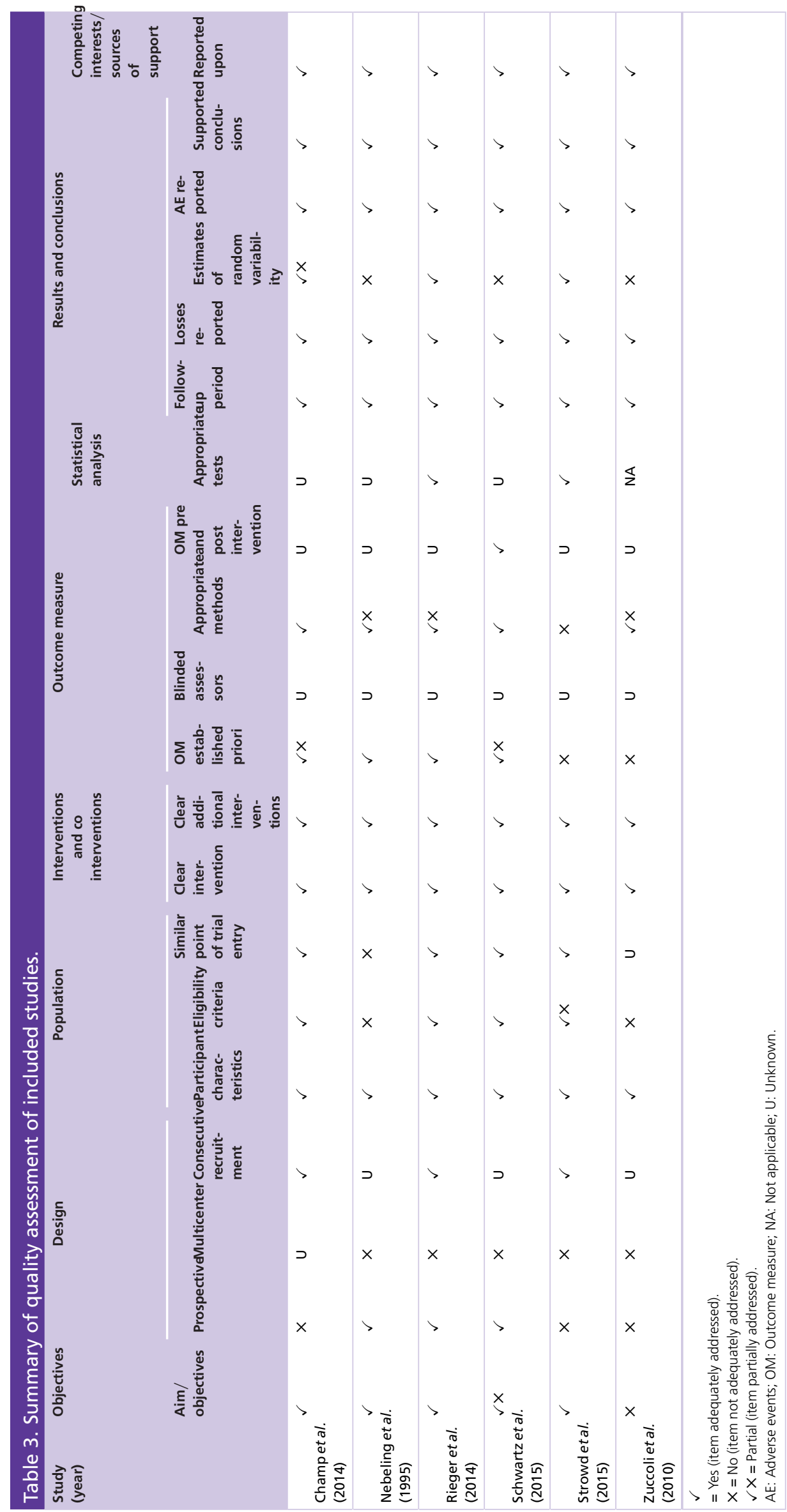




\section{Glucose levels}

Five studies reported serum glucose levels $(\mathrm{n}=18)$. Three studies $(\mathrm{n}=14)$ reported a decrease in serum glucose during diet compared with prediet levels. However, levels varied from a mean nonfasting serum glucose of $7.9 \mathrm{mmol} / \mathrm{l}$ (indicates reported units have been converted to $\mathrm{mmol} / \mathrm{l}$ from $\mathrm{mg} / \mathrm{dl}$ for comparison) prediet (no SD) to $4.7 \mathrm{mmol} / \mathrm{l}$ (indicates reported units have been converted to $\mathrm{mmol} / \mathrm{l}$ from $\mathrm{mg} / \mathrm{dl}$ for comparison) (no SD; $\mathrm{n}=4$ ) [21], to $7.5 \mathrm{mmol} / \mathrm{l}$ prediet decreasing to $3.5 \mathrm{mmol} / \mathrm{l}$ during diet $(\mathrm{n}=1)$ [25], with a less extreme response noted in one study $(5.5 \pm 1.2 \mathrm{mmol} / \mathrm{l}$ [indicates reported units have been converted to $\mathrm{mmol} / \mathrm{l}$ from $\mathrm{mg} / \mathrm{dl}$ for comparison] prediet to $5.1 \pm 0.5 \mathrm{mmol} / \mathrm{l}$ [indicates reported units have been converted to $\mathrm{mmol} / \mathrm{l}$ from $\mathrm{mg} / \mathrm{dl}$ for comparison] during diet; $n=9)$ [22]. One study reported serum glucose levels during diet only $(3.5-5.5 \mathrm{mmol} / \mathrm{l} ; \mathrm{n}=2)$ [26] and one study reported serum glucose could not be maintained below the target of $4.4 \mathrm{mmol} / 1$ [indicates reported units have been converted to $\mathrm{mmol} / \mathrm{l}$ from $\mathrm{mg} / \mathrm{dl}$ for comparison] $(\mathrm{n}=2)$ [24].

\section{Ongoing trials \\ Results of search}

Eighteen records of ongoing trials were identified within study registries. After removing duplicates, 12 records relating to 12 individual trials remained (total participant population of $\mathrm{n}=265$ ), all of which were eligible for this review [27-38]. Table 4 summarizes characteristics of the 12 ongoing clinical trials.

\section{Discussion}

Summary of main results

This systematic review identified no high quality prospective studies assessing KD for glioma, but did identify a number of small randomized controlled trials that are currently ongoing. All six published case series included in this review reported overall or PFS; however due to the limited sample sizes (ranging from one to twenty participants) and the absence of a control group, it is not possible to make any conclusion as to the effectiveness of the KD interventions.

Adverse events were consistent across the majority of studies, predominately being weight loss and raised cholesterol. However, two studies adopted an energy restricted KD, following which weight loss would be expected [24,25]. The significance and clinical impact of weight loss would need to be considered and could be managed through nonenergy restricted, nonfasting regimes supported by a trained dietitian [26]. The impact of KD on cholesterol profiles should also be considered within the context of a disease that has poor long-term survival. While two studies reported an increase in cholesterol [24,26], one study, conflictingly reported cholesterol to reduce over the course of the diet [22]; therefore requires further investigation.

Retention rates on diet varied from 50 to $100 \%$, however only three studies utilized a study protocol with predetermined duration for the dietary intervention [22,24,26]. As sample sizes of these studies range from 2 to 20 participants, the external validity of such data is questionable. No studies reported quality of life or dietary acceptability using the appropriate objective or subjective measures and are therefore subject to performance bias. Future studies should consider the inclusion of validated measures to assess quality of life and dietary acceptability.

Dietary compliance was inconsistently measured, with two studies citing the presence of serum ketones as a marker of compliance [24,26], and one study using self-reported measures [22]. Both methods have their limitations; including selection bias with eligibility criteria requiring patients to be compliant with the diet prior to recruitment [24] and reporter bias from self-reported measures [22]. Due to diverse methodologies, it is not possible to determine which diet is easier for participants to comply with.

A trend for the decrease in serum glucose levels, while adhering to a $\mathrm{KD}$, can be noted across the studies, with glucocorticoids having a negative impact on levels. However, the clinical impact of this cannot be determined from the results of the studies so far. Five studies measured ketones in either urine or serum; however due to different methods no comparisons can be made between diets.

This review also identified 12 ongoing studies, five of which are randomized control trials (RCT). Three RCTs may be suitable for future meta-analysis [28-29,31]. These studies have comparable populations, outcome measures, control groups and similar dietary interventions, but further dietary and methodological details would be required to assess the appropriateness of such an analysis. The planned recruitment figures remain small and the trials are underpowered to demonstrate effectiveness. A multicenter RCT or the ability to undertake a meta-analysis is required. 


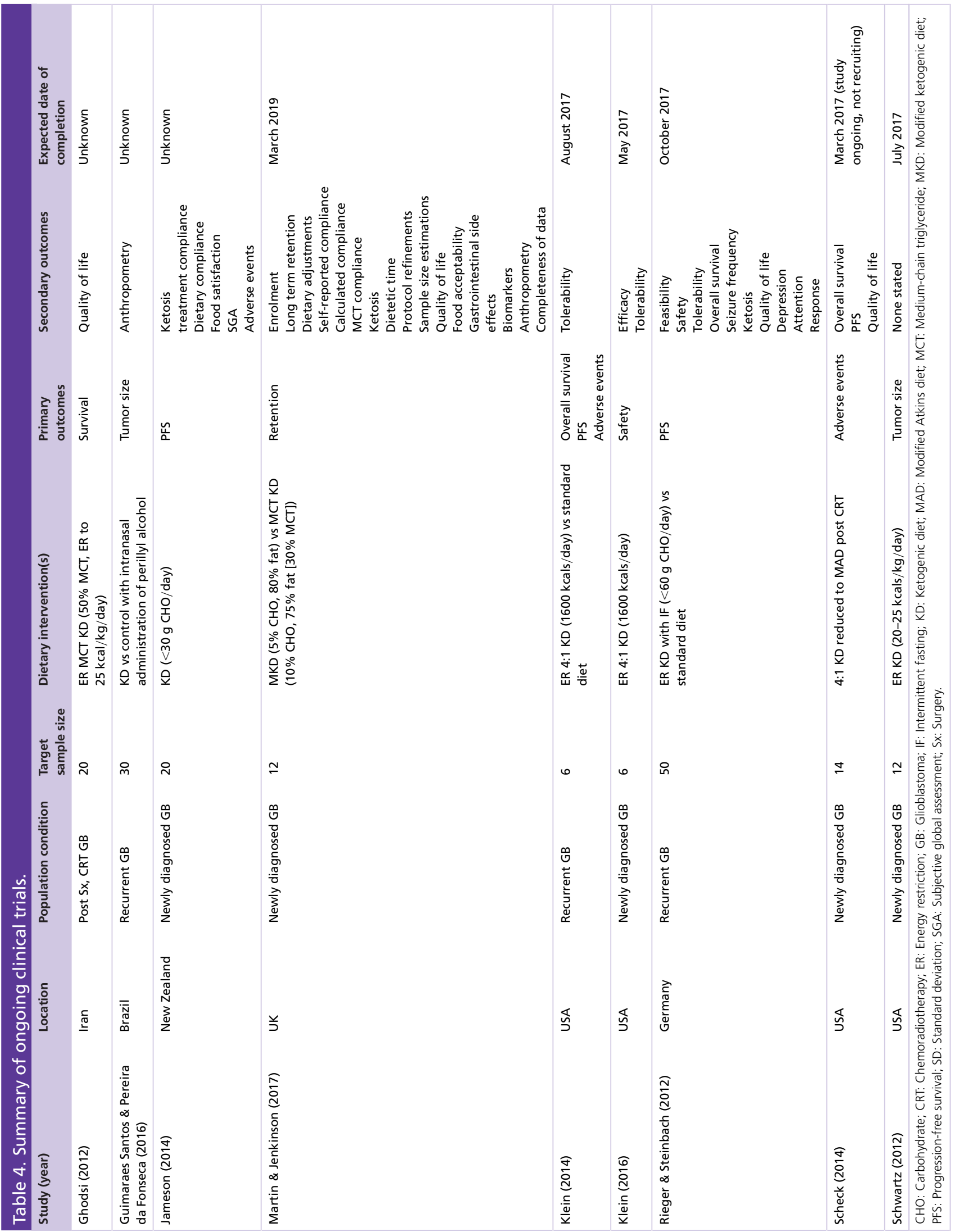




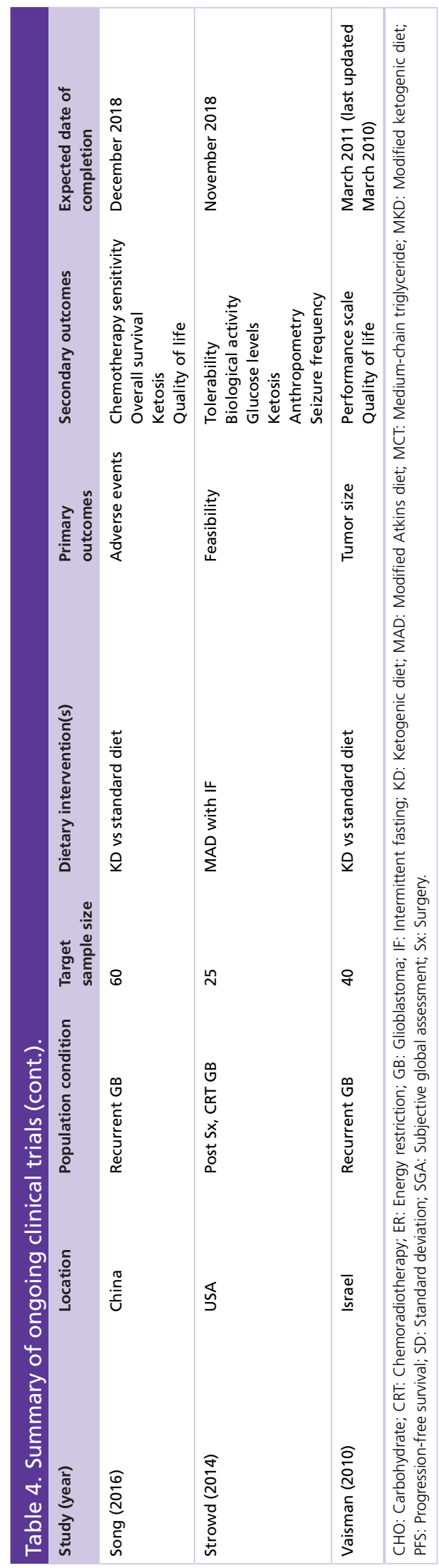




\section{Potential biases in the review process}

Thorough database searches were performed to identify studies suitable for this review. Application of the eligibility criteria to the research results identified six studies for inclusion. Given the relative novelty of $\mathrm{KD}$ in gliomas, a low number of editorials were expected. As the search strategy was first piloted and the results of the search strategy supplemented by hand searches, it is unlikely that relevant studies were missed, further confirmed through contact with an expert in the field. Therefore conclusions drawn from the review are based upon all available evidence.

A key strength of this review lies in the quality assessment of included studies. The Institute of Health Economics (IHE; Canada) Case Series Quality Appraisal Checklist [9] is the only validated quality appraisal tool for assessing the methodological quality of case series $[9,39]$. The tool was updated to include assessor annotations specific to this review as recommended by the authors of the tool [9], to aid the quality appraisal process. The tool does not provide a scoring system in which study quality may be distinguished as high or low, therefore it was not possible to strictly assess the confidence of cumulative evidence. As such, a narrative approach was taken in this review. No studies fulfilled the full study design criteria of the quality assessment tool.

Three studies utilized a study protocol enabling the repetition of their methods $[22,24,26]$. Of these, one study author [24] provided the trial protocol and there appeared to be no suggestion of selective reporting bias.

One study included a retrospective control group [21], however failed to statistically or descriptively compare the control group to the dietary intervention group. The control group was also unlikely to represent the population due to convenience sampling methods and eligibility criteria requiring variables not held within the records, thus creating selection bias. Therefore, this study design was also considered a case series.

A meta-analysis was not undertaken due to the heterogeneity of the study populations, methods, outcomes and bias stated previous. The overall measure of treatment effect would be misleading given studies were not powered to determine treatment effect. Study populations varied from newly diagnosed grade III or IV gliomas to recurrent gliomas and the KD was administered at different time points in the treatment pathway. Participants also received a wide variety of oncology treatments while following $\mathrm{KD}$. The KD interventions also varied, in terms of energy and carbohydrate restrictions, and types of dietary fats included. One study included fermented yoghurts and plant oils in addition to $\mathrm{KD}$ [22], therefore presenting difficulties when comparing $\mathrm{KD}$ outcomes. Ongoing studies may provide better quality data and synergy between protocols to allow for meta-analysis in future reviews.

\section{Implications for practice \& research}

Due to the lack of high quality evidence it is difficult to justify the use of KDs in a clinical, nonresearch setting. Further research is required to explore dietary acceptability, cost effectiveness and clinical effectiveness, prior to implementation alongside the current standard of care in this population.

Key areas for future research include:

- A pragmatic feasibility study to inform future RCT design; with outcomes related to adverse events, retention rates, quality of life, dietary acceptability, tolerability and compliance, using validated measures, as adopted by the KEATING study [32].

- Determining if KDs are effective in the management of glioma, through high quality RCTs. It will be important to consider which $\mathrm{KD}$, if any, is beneficial and at what point in the treatment pathway. $\mathrm{KD}$ concurrent to chemoradiotherapy in animal models has proven to potentiate the treatment effects; however, this is yet to be replicated within glioblastoma patients. Testing clinical effectiveness for median OS, in a newly diagnosed glioblastoma population, would require approximately 600 participants and is therefore unlikely to be achieved. It may be practical to power studies to PFS or OS at 6 months to enable attainable recruitment figures.

- A health economic assessment to establish efficiency, clinical effectiveness and value of the intervention, would be beneficial. Establishing quality adjusted life years would be of benefit to assess disease burden, in terms of quality and quantity of life gained by patients, if at all any.

\section{Conclusion}

This review is based on case series evidence, the lowest position in the evidence hierarchy. However, at present this is the only evidence available to inform decisions regarding the implementation of KDs for gliomas. While the review has found minimal adverse events, suggesting KDs to be safe in this population, the evidence for effectiveness and acceptability of various KDs is insufficient to suggest they have a therapeutic effect in the management of gliomas. Further high-quality research would be of benefit. 


\section{Future perspective}

Over the next 5 to 10 years we may see the field of KDs in gliomas expanding, following the publication of current ongoing studies. There will be the opportunity for meta-analysis presenting more robust evidence on the subject. However, it may be several years before large scale, RCT evidence is published exploring the effectiveness of KDs in gliomas.

\section{Supplementary data}

To view the supplementary data that accompany this paper please visit the journal website at: www.futuremedicine.com/doi/full/ $10.2217 /$ cns-2017-0030

\section{Author's contributions}

KJ Martin-McGill designed and wrote the manuscript. MD Jenkinson, N Srikandarajah, AG Marson and CT Smith reviewed manuscript with suggestions. N Srikandarajah participated in data extraction and quality assessment.

\section{Acknowledgements}

The authors would like to thank AC Scheck, Associate Professor, Dignity Health, Phoenix, USA for providing her expert opinion on pertinent studies for inclusion in this review.

Financial \& competing interests disclosure

MD Jenkinson, AG Marson, C Tudur Smith, KJ Martin-McGill receive funding from Vitaflo (International) Ltd for KJ Martin-McGill PhD studies. However, Vitaflo did not contribute to or influence this review. The authors have no other relevant affiliations or financial involvement with any organization or entity with a financial interest in or financial conflict with the subject matter or materials discussed in the manuscript apart from those disclosed.

Writing assistance was utilized in the production of this manuscript - funded by Vitaflo (International) Ltd.

\section{Open access}

This work is licensed under the Creative Commons Attribution 4.0 License. To view a copy of this license, visit http://creativecomm ons.org/licenses/by/4.0/

PROSPERO protocol registration number

CRD42017056752.

\section{References}

1. Ostrom QT, Gittleman H, Fulop J et al. CBTRUS statistical report: primary brain and central nervous system tumors diagnosed in the United States in 2008-2012. Neuro Oncol. 17(Suppl. 4), IV1-IV62 (2015).

2. Stupp R, Brada M, van den Bent M, Tonn J, Pentheroudakis G. High-grade glioma: ESMO Clinical Practice Guidelines for diagnosis, treatment and follow-up. Ann. Oncol. 25(Suppl. 3), III93-III101 (2014).

3. Warburg O, Wind F, Negelein E. The metabolism of tumors in the body. J. Gen. Physiol. 309(2), 397-519 (1926).

4. Maurer GD, Brucker DP, Bähr O et al. Differential utilization of ketone bodies by neurons and glioma cell lines: a rationale for ketogenic diet as experimental glioma therapy. BMC Cancer 11(1), 315 (2011).

5. Seyfried TN, Mukherjee P. Targeting energy metabolism in brain cancer: review and hypothesis. Nutr. Metab. 2, 30 (2005).

6. Martin K, Jackson CF, Levy RG, Cooper PN. Ketogenic diet and other dietary treatments for epilepsy. Cochrane Database Syst. Rev. 2, CD001903 (2016).

7. Klement RJ, Champ CE, Otto C, Kämmerer U. Anti-tumor effects of ketogenic diets in mice: a meta-analysis. PLoS ONE 11(5), 1-16 (2016).

8. Moher D, Liberati A, Tetzlaff J, Altman DG. Preferred reporting items for systematic reviews and meta-analyses: the PRISMA statement. BMJ 339, b2535 (2009).

9. Guo B, Moga C, Harstall C, Schopflocher D. A principal component analysis is conducted for a case series quality appraisal checklist. J. Clin. Epidemiol. 69, 199.e2-207.e2 (2016).

10. Brooks KS, Woolf EC, Scheck AC. The ketogenic diet as an adjuvant therapy for brain tumors and other cancers. In: Critical Dietary Factors in Cancer Chemoprevention. Ullah MF, Ahmad A (Eds). Springer International Publishing, Cham, Switzerland, 89-109 (2016).

11. Cohen JE, Wakefield CE, Cohn RJ. Nutritional interventions for survivors of childhood cancer. Cochrane Database Syst. Rev. 8, CD009678 (2016). 
12. Dang MT, Wehrli S, Dang CV, Curran T. The ketogenic diet does not affect growth of hedgehog pathway medulloblastoma in mice. PLoS ONE 10(7), e0133633 (2015).

13. Fine EJ, Segal-Isaacson CJ, Feinman RD et al. Targeting insulin inhibition as a metabolic therapy in advanced cancer: a pilot safety and feasibility dietary trial in 10 patients. Nutrition 28(10), 1028-1035 (2012).

14. Schmidt M, Pfetzer N, Schwab M, Strauss I, Kämmerer U. Effects of a ketogenic diet on the quality of life in 16 patients with advanced cancer: a pilot trial. Nutr. Metab. (Lond.) 8(1), 54 (2011).

15. Seyfried BTN, Kiebish M, Marsh J, Mukherjee P. Targeting energy metabolism in brain cancer through calorie restriction and the ketogenic diet. J. Cancer Res. Ther. 5(9), 7-15 (2009).

16. Nebeling LC, Lerner E. Implementing a ketogenic diet based on medium-chain triglyceride oil in pediatric patients with cancer. J. Am. Diet. Assoc. 95(6), 693-697 (1995).

17. Artzi M, Liberman G, Vaisman $\mathrm{N}$ et al. Changes in cerebral metabolism during ketogenic diet in patients with primary brain tumors: 1H-MRS study. J. Neurooncol. 132(2), 267-275 (2017).

18. Shen V, Tran L, Brown J. Use of ketogenic diet as a complementary metabolic therapy during chemo-raditation in a 7 year old female with glioblastoma. Neuro Oncol. 18(Suppl. 3), iii54 (2016).

19. Kalamian M, Zupec-Kania B, Favara BE, Liepa GU. Ketogenic diet as adjunctive therapy for brain tumors. Poster presented at: The First International Dietary Treatments for Epilepsy and other Neurologic Disorders. Phoenix, Arizona, 2-5 April 2008.

20. Attar H, Rolfe S, Marsey M, Rogers L. Results of the modified atkins diet in patients with recurrent glioma: retrospective review. Neurology 86(16), P4.246 (2016).

21. Champ CE, Palmer JD, Volek JS et al. Targeting metabolism with a ketogenic diet during the treatment of glioblastoma multiforme. J. Neurooncol. 117(1), 125-131 (2014).

22. Rieger J, Bähr O, Maurer GGD et al. ERGO: a pilot study of ketogenic diet in recurrent glioblastoma. Int. J. Oncol. 45(6), 1843-1852 (2014).

23. Strowd RE, Cervenka MC, Henry BJ, Kossoff EH, Hartman AL, Blakeley JO. Glycemic modulation in neuro-oncology: experience and future directions using a modified Atkins diet for high-grade brain tumors. Neuro Oncol. Pract. 2(3), 127-136 (2015).

24. Schwartz K, Chang HT, Nikolai M et al. Treatment of glioma patients with ketogenic diets: report of two cases treated with an IRB-approved energy-restricted ketogenic diet protocol and review of the literature. Cancer Metab. 3(3), 1-10 (2015).

25. Zuccoli G, Marcello N, Pisanello A et al. Metabolic management of glioblastoma multiforme using standard therapy together with a restricted ketogenic diet: case report. Nutr. Metab. (Lond.) 7(33), 1-7 (2010).

26. Nebeling LC, Miraldi F, Shurin S, Lerner E. Effects of a ketogenic diet on tumor metabolism and nutritional status in pediatric oncology patients: two case reports. J. Am. Coll. Nutr. 14(2), 202-208 (1995).

27. Clinical trials database: NCT01865162. https://clinicaltrials.gov/ct2/show/NCT01865162?term=ketogenic+diet+and+GBM\&rank=1

28. Clinical trials database: NCT02939378. https://clinicaltrials.gov/ct2/show/NCT02939378?term=ketogenic+diet+and+gbm\&rank=2

29. Clinical trails database: NCT02302235. https://clinicaltrials.gov/ct2/show/NCT02302235?term=ketogenic+diet+and+gbm\&rank=3

30. Clinical trials database: NCT02046187. https://clinicaltrials.gov/ct2/show/study?term=ketogenic+diet+and+gbm\&rank=4

31. Clinical trials database: NCT01754350. https://clinicaltrials.gov/ct2/show/NCT01754350

32. Clinical trials database: NCT03075514. https://clinicaltrials.gov/ct2/show/NCT03075514

33. Clinical trails database: NCT01535911. https://clinicaltrials.gov/ct2/show/NCT01535911

34. Clinical trials database: NCT01092247. https://clinicaltrials.gov/ct2/show/NCT01092247

35. Clinical trials database: NCT02286167. https://clinicaltrials.gov/ct2/show/NCT02286167

36. Ghodsi SM. Therapeutic effect of ketogenic diet on survival and quality of patients with Glioblastoma Multiforme. IRCT identifier: IRCT201204099417N1 (2012). http://apps.who.int/trialsearch/Trial2.aspx?TrialID=IRCT201204099417N1

37. Jameson M. Pilot study evaluating progression-free survival in patients using a ketogenic diet while receiving chemoradiation for glioblastoma multiforme. ANZCTR identifier:

ACTRN12614001056684 (2014). http://apps.who.int/trialsearch/Trial2.aspx?TrialID=ACTRN12614001056684

38. Guimaraes Santos J, Pereira da Fonseca CO. Ketogenic diet combined with intranasal administration of perillyl alcohol: strategy therapy to refractory Glioblastoma Multiforme to standard treatment. REBEC identifier: RBR-8x8fd9 (2016). http://apps.who.int/trialsearch/Trial2.aspx?TrialID=RBR-8x8fd9

39. Moga C, Guo B, Harstall C. Development of a Quality Appraisal Tool for Case Series Studies Using a Modified Delphi Technique. Institute of Health Economics, Alberta, Canada, 1-71 (2012). 
